\title{
Hill 屈服准则向拉-压非对称材料的推广
}

\author{
陈雷，温卫东"，崔海涛 \\ 南京航空航天大学能源与动力学院, 南京 210016 \\ *E-mail: gswwd@nuaa.edu.cn
}

收稿日期: 2012-07-04; 接受日期: 2012-08-20

\begin{abstract}
摘要本文旨在建立一个形式简单、能同时描述金属的塑性各向异性及拉-压非对称性的 宏观屈服准则。首先，巧妙地构造了一个与静水压力无关，能描述金属材料拉一压非对称性 的宏观各向同性屈服准则; 其次, 利用 Cazacu 的广义应力偏量不变量, 将该准则推广到正 交各向异性条件，使其能同时描述金属的塑性各向异性及拉-压非对称性. 该准则形式简 单, 待定材料常数只有 $\mathrm{Cazacu}$ 提出的同类屈服准则的一半, 更便于实际应用. 对比拉-压对 称的 Hill (1948) 屈服准则, 本文提出的准则增加了 3 个描述材料拉-压非对称性的材料常数, 所有常数都可以通过简单试验确定. 当材料拉-压性能相同时, 该准则退化为 Hill (1948)屈 服准则. 因此，本文提出的准则可以看作 Hill (1948)屈服准则向拉-压非对称材料的推广. 对 $\mathrm{Cu}-\mathrm{Al}-\mathrm{Be}$ 记忆合金(试验数据来自 Laydi 和 Lexcellent)及 $\mathrm{Ni}_{3} \mathrm{Al}$ 基金属间化合物 IC10 合 金的应用表明，本文提出的屈服准则能很好地描述这两种合金的塑性各向异性及拉-压非 对称性.
\end{abstract}

关键词

塑性各向异性

孪生

拉-压非对称性

屈服准则

记忆合金

金属间化合物
金属材料在复杂应力状态下的屈服准则是塑性 力学研究中最基础的内容, 它在物理、力学、材料科 学等领域得到广泛应用. 屈服准则的研究在金属塑 性成形及有效利用材料方面具有非常重要的意义 ${ }^{[1,2]}$. 因此, 关于材料屈服准则的研究一直是力学领域一 个非常重要的课题 ${ }^{[3]}$. 半个世纪以来, 各国学者在材 料屈服准则方面做了大量工作. 其中引人注目的工 作由 Hill ${ }^{[1 \sim 6]}$, Hosford ${ }^{[7,8]}$, Barlat 等人 ${ }^{[9 \sim 11]}$ 做出.

对于金属材料, 其主要塑性变形机制是位错滑 移和孪生(某些特定金属)。不管是位错还是孪生，相 应的剪切变形都发生在特定的晶面, 沿着特定的方 向. 假如材料通过可逆的切变机制进行塑性变形, 则 屈服与剪应力的符号无关, 只与滑移面滑移方向的 分解剪应力大小有关, 其拉伸屈服强度与压缩屈服
强度大小相等. 大多数屈服准则是基于拉-压对称假 设建立的, 其屈服函数是应力偏量的偶函数. 然而, 并非所有金属都是拉-压对称的. 具有基面或准基面 织构的密排六方 (hcp)金属, $\{10 \overline{1} 2\}$ 孪生在面内压缩 时很容易被激活，在拉伸时则不会被激活. 由于这种 与方向相关的孪生, 材料在低应变水平下具有非常 显著的拉-压非对称性(SD 效应 $)^{[12,13]}$. 一般而言, 其 压缩屈服强度远远低于拉伸屈服强度. 另外, $\mathrm{LI}_{2}$ 型长 程有序金属间化合物由于其不满足 Schmid 定律的晶 体滑移而具有明显的拉-压非对称性 ${ }^{[14,15]}$. 金属材料 的屈服通常与静水压力无关, 即叠加静水压力不会 引起材料的屈服. 为了建立一个与静水压力无关, 适 合金属材料的拉-压非对称屈服准则, Hosford $(1966)^{[7]}$ 将 Hill(1948)准则 ${ }^{[4]}$ 改进为以下形式:

英文版发表信息： Chen L, Wen W D, Cui H T. Generalization of Hill's yield criterion to tension-compression asymmetry materials. Sci China Tech Sci, 2012, 55: 89-97, doi: 10.1007/s11431-012-5037-9 


$$
\begin{aligned}
& F\left(\sigma_{y y}-\sigma_{z z}\right)^{2}+G\left(\sigma_{z z}-\sigma_{x x}\right)^{2}+H\left(\sigma_{x x}-\sigma_{y y}\right)^{2} \\
& +A \sigma_{x x}+B \sigma_{y y}+(-B-A) \sigma_{z z}=1,
\end{aligned}
$$

式中 $x, y, z$ 分别与材料 3 个各向异性主轴重合. $F, G$, $H, A, B$ 为材料常数, $\sigma_{z z}$ 的系数不独立是为了满足屈 服与静水压力无关这一条件. 该准则的屈服面在三 维主应力空间为轴线与静水应力轴 $\left(\sigma_{x x}=\sigma_{y y}=\sigma_{z z}\right)$ 平 行的无限椭圆柱面; 其在 $\pi$ 平面 (与静水应力轴垂直 的平面)上的椭圆圆心由增加的系数 $A, B$ 确定. Hosford (1966) ${ }^{[7]}$ 准则能准确描述 SD 效应较弱、具有 椭圆形屈服轨迹的 hcp 金属(如钛合金)的屈服行为. 然而, 该准则不包含剪应力且存在理论上的缺陷. 假 设材料主轴 $x, y$ 方向的拉伸屈服强度大于压缩屈服 强度, 则可以得出 $A<0, B<0 ;$ 从而 $(-B-A)>0$, 得出材 料主轴 $z$ 方向的拉伸屈服强度小于压缩屈服强度的不 合理结论. 这是由该准则屈服面的平移(从而描述 SD 效应)导致的, 图 1 演示了这一过程. 因此简单加入正 应力的线性项无法建立一个理论完备且与静水压力 无关的屈服准则, 为此需要寻求应力的更高次项.

最近, Cazacu 和 Barlat ${ }^{[16]}$ 提出了一个能描述金 属材料 SD 效应的各向同性屈服准则, 并利他们提出 的广义应力偏量不变量 ${ }^{[17,18]}$ 将其推广到正交各向异 性条件. Cazacu 屈服准则(详细表达式见附录 A)用于 3-D 应力状态时包含 18 个材料常数; 平面应力状态 时 $\left(\sigma_{x x}, \sigma_{y y}, \sigma_{x y}\right)$ 包含 11 个材料常数. Cazacu 将该准则 应用到 $\mathrm{Mg}, \mathrm{Mg}-\mathrm{Th}$ 和 $\mathrm{Mg}-\mathrm{Li}$ 合金, 预测结果与试验数

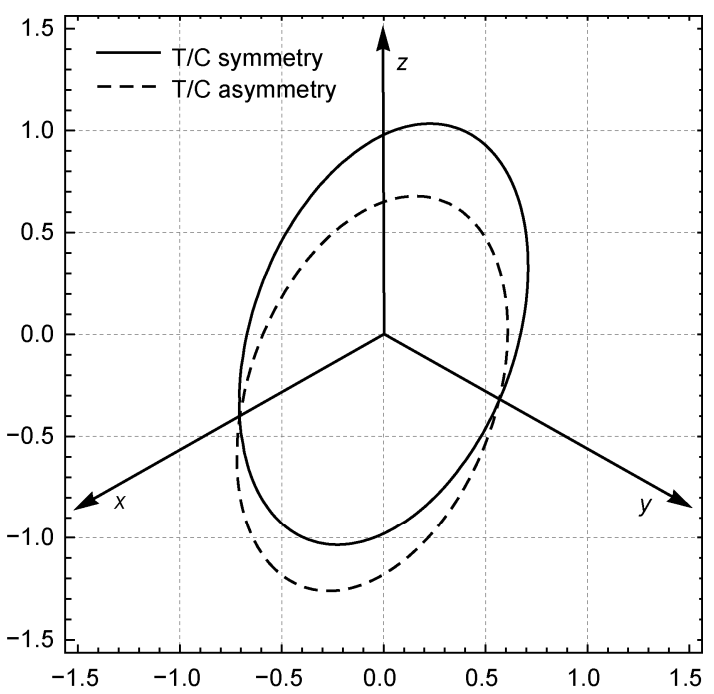

图 1 Hosford $(1966)^{[7]}$ 准则屈服面在 $\pi$ 平面上的投影
据吻合得很好. Cazacu 准则能很好地描述 hcp 金属的 塑性各向异性及 SD 效应, 引起了广泛关注 ${ }^{[19 ~ 23]}$. 此 后, 各国学者提出了一系列能描述 SD 效应的各向异 性屈服准则 ${ }^{[24,25]}$. 然而, 这些屈服准则要么形式过于 复杂, 要么包含过多材料常数, 不便于实际应用.

Hill (1948) 屈服准则 ${ }^{[4]}$ 是具有里程碑意义的一个 准则, 它的有效性被很多试验证实. 由于它简洁对称, 根据流动法则导出的线性塑性应变-应力关系给计算 带来很大方便, 至今仍是应用最广的屈服准则之一. $\mathrm{Hill}^{[5]}$ 曾指出, 对材料屈服准则的改进不应以牺牲过 多的简洁性为代价。正是基于便于实际应用这一朴 素思想, 本文尝试建立一个形式简单, 包含最少材料 常数, 且预测能力较强的拉-压非对称屈服准则. 首 先构造一个与静水压力无关、能描述金属拉-压非对 称性(SD 效应)的宏观各向同性屈服准则; 其次利用 Cazacu 的广义应力偏量不变量 ${ }^{[17,18]}$, 将其推广到正 交各向异性条件，建立一个能同时描述金属塑性各 向异性及 SD 效应的屈服准则; 最后用建立的屈服准 则对 $\mathrm{Cu}-\mathrm{Al}-\mathrm{Be}$ 记忆合金(试验数据来自 Laydi 和 Lexcellent ${ }^{[26]}$ )及 $\mathrm{Ni}_{3} \mathrm{Al}$ 基金属间化合物 IC10 合金在平 面应力状态下的屈服行为进行预测.

\section{1 与静水压力无关的拉-压非对称各向同 性屈服准则}

对于各向同性屈服准则, 应用最广的是 Tresca 最大剪应力准则和 von Mises 畸变能准则. Tresca 准 则认为中间主应力对材料的屈服没有影响; von Mises 准则认为 3 个主应力对材料的屈服具有相同影 响. 除了这两个著名的准则, 很多学者提出了介于两 者之间的屈服准则. Hershey ${ }^{[27]}$ 提出了一个指数 $a$ 可 变的屈服准则, 该准则后来被 Hosford ${ }^{[8]}$ 推广到正交 各向异性条件. 当指数 $a$ 取不同值时, Hershey 准则可 以得到从 Tresca 到 von Mises 的一族屈服面. 我国学 者俞茂宏提出的双剪应力系列理论 ${ }^{[28,29]}$ 同时考虑了 最大剪应力和中间剪应力对材料屈服的影响, 可用 于描述多种材料的屈服行为, 得到广泛应用. 最近, 姚仰平等人 ${ }^{[30]}$ 提出了一个广义非线性强度理论, 用 一个表达式描述了 $\pi$ 平面及子午面上各种材料的非 线性强度特性, 能很好地描述混凝土和堆石材料的 破坏特性.

Drucker $^{[31]}$ 提出了一个同时包含两个应力偏量不 
变量的屈服准则:

$$
J_{2}^{3}-c J_{3}^{2}=\tau_{\mathrm{Y}}^{6},
$$

其中 $J_{2}=\operatorname{tr} \boldsymbol{S}^{2} / 3, J_{3}=\operatorname{tr} \boldsymbol{S}^{3} / 3$ 分别为应力偏量 $\boldsymbol{S}$ 的 第二、第三不变量 $\left(\operatorname{tr}\right.$ 表示迹运算 $\left.\operatorname{tr}(\boldsymbol{A})=\sum_{k=1}^{3} A_{k k}\right) ; \tau_{\mathrm{Y}}$ 为材料剪切屈服强度. 为了满足屈服面外凸性, $c \in[-27 / 8,2.25]$. 通过 $c$ 的选择, 可以很好地权衡 3 个主应力对材料屈服的影响. 图 2 所示为 von Mises, Tresca 和 Drucker 屈服准则 ( $c=2.25$ ) 在平面应力状态 下的屈服轨迹. 当 $c=2.25$ 时, Drucker 屈服准则的理 论屈服轨迹与 $24 \mathrm{ST}$ 铝合金试验数据吻合很好.

为了描述 hcp 金属的 $\mathrm{SD}$ 效应, Cazacu 和 Barlat ${ }^{[16]}$ 改进了 Drucker 屈服准则((2)式), 提出了以下准则:

$$
\left(J_{2}\right)^{3 / 2}-c J_{3}=\tau_{\mathrm{Y}}^{3} .
$$

并将其推广到正交各向异性条件，建立了一个 能同时描述材料塑性各向异性及拉-压非对称性的宏 观屈服准则:

$$
\left(J_{2}^{0}\right)^{3 / 2}-c J_{3}^{0}=\tau_{\mathrm{Y}}^{3},
$$

其中 $\tau_{\mathrm{Y}}$ 为剪切屈服强度, $c$ 为材料常数; $J_{2}^{0}, J_{3}^{0}$ 分别 为应力偏量 $\boldsymbol{S}$ 第二、第三不变量 $J_{2}, J_{3}$ 在正交各向异 性条件下的推广. 该准则用于 3-D 应力状态时包含 18 个材料系数, 平面应力状态时包含 11 个材料系数 (详细表达式见附录 $\mathrm{A}$ ).

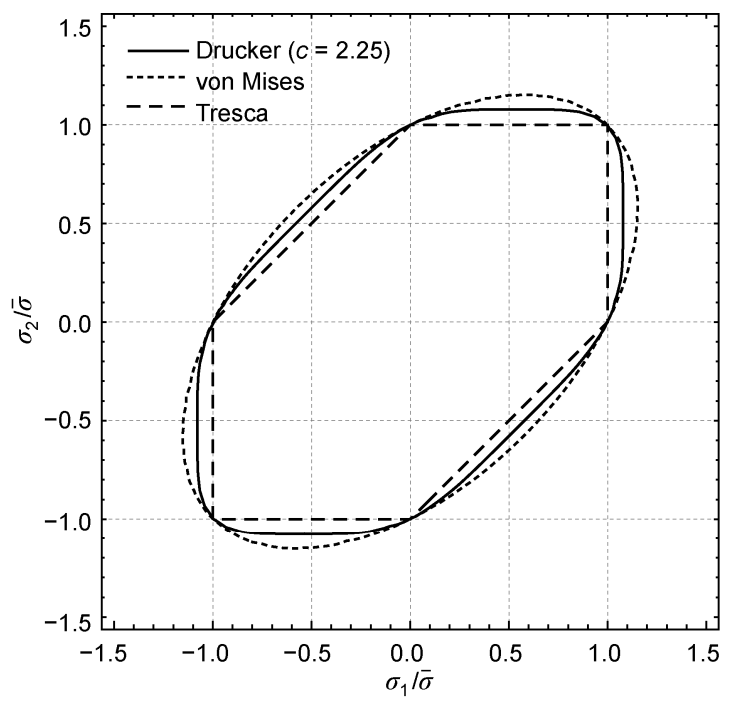

图 2 Drucker, Tresca 和 von Mises 屈服准则在平面应力 状态下的屈服轨迹
考虑到 Cazacu 准则包含过多材料系数, 不便于 实际应用. 下面构造一个形式简单、能描述材料 SD 效应的各向同性屈服准则, 并在下节将其推广到正 交各向异性条件.

受 Cazacu 等人 ${ }^{[16]}$ 的启发, 本文构造了一个能描 述 SD 效应的各向同性屈服准则, 当坐标系与应力主 轴重合时, 具有以下形式:

$$
\begin{aligned}
f= & f\left(\sigma_{i j}\right)=\left(J_{2}\right)^{3 / 2}-c\left\{2\left(\sigma_{x x}^{3}+\sigma_{y y}^{3}+\sigma_{z z}^{3}\right)\right. \\
& -3\left[\left(\sigma_{y y}+\sigma_{z z}\right) \sigma_{x x}^{2}+\left(\sigma_{z z}+\sigma_{x x}\right) \sigma_{y y}^{2}\right. \\
& \left.\left.+\left(\sigma_{x x}+\sigma_{y y}\right) \sigma_{z z}^{2}\right]+12 \sigma_{x x} \sigma_{y y} \sigma_{z z}\right\} \\
= & Y^{3},
\end{aligned}
$$

其中 $c$ 为材料常数, $Y$ 为材料的拉伸(压缩)屈服强度. 容易证明

$$
f\left(\sigma_{i j}+\sigma_{m}\right)=f\left(\sigma_{i j}\right),
$$

其中 $\sigma_{m}=\left(\sigma_{x x}+\sigma_{y y}+\sigma_{z z}\right) / 3$ 为平均正应力, 这表明屈服 准则(5)与静水压力无关.

引入应力偏量, (5)式变为

$$
\begin{aligned}
\varphi= & \left(J_{2}\right)^{3 / 2}-c\left\{2\left(S_{x}^{3}+S_{y}^{3}+S_{z}^{3}\right)-3\left[\left(S_{y}+S_{z}\right) S_{x}^{2}\right.\right. \\
& \left.\left.+\left(S_{z}+S_{x}\right) S_{y}^{2}+\left(S_{x}+S_{y}\right) S_{z}^{2}\right]+12 S_{x} S_{y} S_{z}\right\} \\
= & Y^{3},
\end{aligned}
$$

其中 $S_{i}, i=x, y, z$ 为应力偏量 $S$ 的分量. 考虑到 $S_{x}+S_{y}+S_{z}=0$, (7) 式可以简化为

$$
\varphi=\left(J_{2}\right)^{3 / 2}-c\left[5\left(S_{x}^{3}+S_{y}^{3}+S_{z}^{3}\right)+12 S_{x} S_{y} S_{z}\right]=Y^{3} .
$$

材料常数 $c$ 的物理意义可以通过单向加载试验得出. 考虑单向拉伸的情况, 并设 $\sigma_{\mathrm{t}}$ 为材料的拉伸屈服强度, 由(8)式

$$
\sigma_{\mathrm{t}}=Y\left(\frac{3 \sqrt{3}}{1-6 \sqrt{3} C}\right)^{1 / 3} .
$$

单向压缩时, 将条件 $\sigma_{1}=-\sigma_{\mathrm{c}}, \sigma_{2}=\sigma_{3}=0$ 带入(8)式 可得

$$
\sigma_{\mathrm{c}}=Y\left(\frac{3 \sqrt{3}}{1+6 \sqrt{3} C}\right)^{1 / 3} .
$$

联立(9)和(10)式

$$
c=\frac{\sqrt{3}}{18} \frac{\left(\sigma_{\mathrm{t}}^{3}-\sigma_{\mathrm{c}}^{3}\right)}{\left(\sigma_{\mathrm{t}}^{3}+\sigma_{\mathrm{c}}^{3}\right)} .
$$

当 $\sigma_{\mathrm{t}}>\sigma_{\mathrm{c}}>0$ 时, $c \in\left(0, \frac{\sqrt{3}}{18}\right)$; 当 $0<\sigma_{\mathrm{t}}<\sigma_{\mathrm{c}}$ 时, 
$c \in\left(-\frac{\sqrt{3}}{18}, 0\right)$. 当材料拉-压性能相同时, $c=0$, 准则(8) 式退化为 von Mises 屈服准则.

在主应力空间中, 准则(8)表示的屈服面是以静 水应力轴为轴线的无限柱面, 且关于原点呈三重旋 转对称. $c \neq 0$ 时, 其在 $\pi$ 平面上的投影为带圆角的“三 角形”.

平面应力状态下, 该准则简化为

$$
\begin{aligned}
& {\left[\frac{1}{3}\left(\sigma_{1}^{2}-\sigma_{1} \sigma_{2}+\sigma_{2}^{2}\right)\right]^{3 / 2}} \\
& -c\left[2 \sigma_{1}^{3}+2 \sigma_{2}^{3}-3\left(\sigma_{1}+\sigma_{2}\right) \sigma_{1} \sigma_{2}\right]=Y^{3} .
\end{aligned}
$$

当 $c \neq 0$ 时, 准则(12)的屈服轨迹为带圆角的“三 角形”. 图 3 所示为拉-压屈服强度比 $\sigma_{\mathrm{t}} / \sigma_{\mathrm{c}}$ 分别为 $4 / 5$, $1,6 / 5$ 时, 方程 (12)确定的屈服轨迹. 相应的材料常数 $c$ 分别为 $-0.031,0$ (von Mises), 0.026 .

\section{2 与静水压力无关的拉-压非对称各向异 性屈服准则}

\section{1 模型的建立}

本节把前面提出的能描述金属材料 SD 效应的各 向同性屈服准则(8)推广到正交各向异性条件, 建立 一个能同时描述材料塑性各向异性及拉-压非对称性

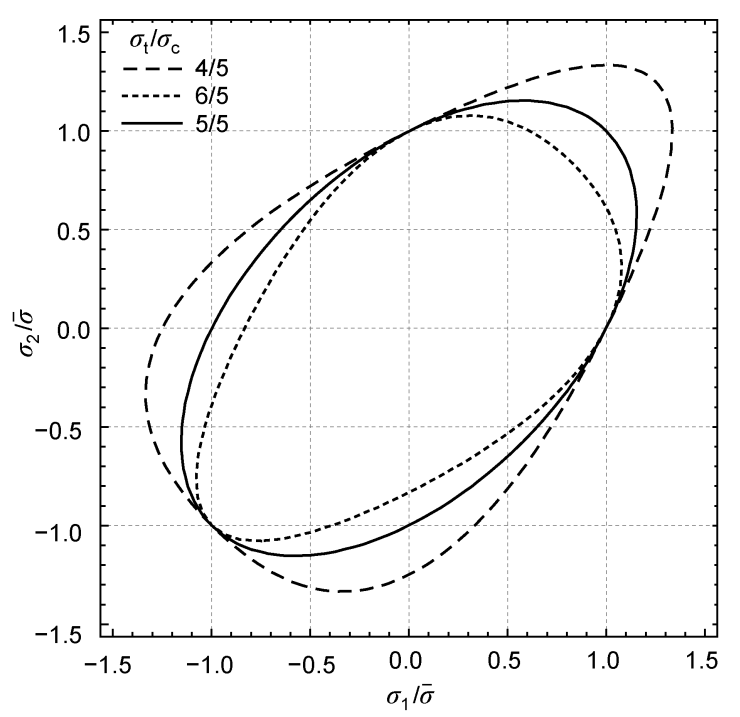

图 3 屈服准则(12)在平面应力状态下的屈服轨迹: $\sigma_{\mathrm{l}} / \sigma_{\mathrm{c}}=$ 4/5, 5/5(von Mises), 6/5 与静水压力无关的拉-压非对称各向同性屈服准则
的屈服准则. $\mathrm{Cazacu}^{[17,18]}$ 提出了一个将应力偏量不变 量推广到各向异性, 从而把相应的各向同性屈服准 则推广到各向异性的一般方法. 用 Cazacu 的广义应 力偏量第二不变量 $J_{2}^{0}$ 代替 $J_{2}$, 并考虑 3 个材料主轴 力学性能的差异, 引入 3 个描述材料拉-压非对称性 的系数 $b_{1}, b_{2}, b_{3}$, 将屈服准则(8)推广到正交各向异性

$$
\begin{aligned}
\Phi= & \left(J_{2}^{0}\right)^{3 / 2}-\left\{5\left(b_{1} S_{x}^{3}+b_{2} S_{y}^{3}+b_{3} S_{z}^{3}\right)\right. \\
& \left.+4\left(b_{1}+b_{2}+b_{3}\right) S_{x} S_{y} S_{z}\right\} \\
= & Y^{3},
\end{aligned}
$$

其中

$$
\begin{aligned}
J_{2}^{0}= & \frac{a_{1}}{6}\left(\sigma_{y y}-\sigma_{z z}\right)^{2}+\frac{a_{2}}{6}\left(\sigma_{z z}-\sigma_{x x}\right)^{2} \\
& +\frac{a_{3}}{6}\left(\sigma_{x x}-\sigma_{y y}\right)^{2}+a_{4} \sigma_{y z}^{2}+a_{5} \sigma_{z x}^{2}+a_{6} \sigma_{x y}^{2},
\end{aligned}
$$

$a_{k}(k=1, \cdots, 6)$ 为描述材料塑性各向异性的常数, $b_{\mathrm{k}}$ $(k=1, \cdots, 3)$ 为描述材料拉-压非对称性的常数, $Y$ 为 材料某一各向异性主轴的屈服强度.

假设 3 个材料主轴的拉伸屈服强度分别为 $\sigma_{1}^{\mathrm{t}}$, $\sigma_{2}^{\mathrm{t}}, \sigma_{3}^{\mathrm{t}}$; 压缩屈服强度分别为 $\sigma_{1}^{\mathrm{c}}, \sigma_{2}^{\mathrm{c}}, \sigma_{3}^{\mathrm{c}} ; 3$ 个材 料对称面对应的剪切屈服强度分别为 $R, S, T$. 将 3 个 材料主轴的单向拉伸(压缩)条件及 3 个材料对称面内 的纯剪条件分别带入(13)式, 可得到确定材料常数 $a_{k}$ $(k=1, \cdots, 6), b_{\mathrm{k}}(k=1, \cdots, 3)$ 的方程组

$$
\begin{gathered}
a_{2}+a_{3}=6\left(\frac{1}{2}\right)^{2 / 3}\left[\left(\frac{Y}{\sigma_{1}^{\mathrm{t}}}\right)^{3}+\left(\frac{Y}{\sigma_{1}^{\mathrm{c}}}\right)^{3}\right]^{2 / 3}, \\
a_{1}+a_{3}=6\left(\frac{1}{2}\right)^{2 / 3}\left[\left(\frac{Y}{\sigma_{2}^{\mathrm{t}}}\right)^{3}+\left(\frac{Y}{\sigma_{2}^{\mathrm{c}}}\right)^{3}\right]^{2 / 3}, \\
a_{1}+a_{2}=6\left(\frac{1}{2}\right)^{2 / 3}\left[\left(\frac{Y}{\sigma_{3}^{\mathrm{t}}}\right)^{3}+\left(\frac{Y}{\sigma_{3}^{\mathrm{c}}}\right)^{3}\right]^{2 / 3}, \\
a_{4}=\frac{Y^{2}}{R^{2}}, \quad a_{5}=\frac{Y^{2}}{S^{2}}, \quad a_{6}=\frac{Y^{2}}{T^{2}}, \\
16 b_{1}+b_{2}+b_{3}=-\frac{9}{2}\left[\left(\frac{Y}{\sigma_{1}^{\mathrm{t}}}\right)^{3}-\left(\frac{Y}{\sigma_{1}^{\mathrm{c}}}\right)^{3}\right], \\
b_{1}+16 b_{2}+b_{3}=-\frac{9}{2}\left[\left(\frac{Y}{\sigma_{2}^{\mathrm{t}}}\right)^{3}-\left(\frac{Y}{\sigma_{2}^{\mathrm{c}}}\right)^{3}\right],
\end{gathered}
$$




$$
b_{1}+b_{2}+16 b_{3}=-\frac{9}{2}\left[\left(\frac{Y}{\sigma_{3}^{\mathrm{t}}}\right)^{3}-\left(\frac{Y}{\sigma_{3}^{\mathrm{c}}}\right)^{3}\right] .
$$

对于横观各向同性材料(如定向凝固合金), 假设 材料主轴 $z$ 为旋转对称轴, 则材料主轴 $x, y$ 方向的力 学性能相同, 有 $a_{1}=a_{2}, b_{1}=b_{2}, a_{4}=a_{5}$, 准则(13)的系数 减少为 6 个. 设 $a_{1}=a_{2}=a_{0}, b_{1}=b_{2}=b_{0}, \sigma_{1}^{\mathrm{t}}=\sigma_{2}^{\mathrm{t}}=\sigma_{0}^{\mathrm{t}}$, $\sigma_{1}^{\mathrm{c}}=\sigma_{2}^{\mathrm{c}}=\sigma_{0}^{\mathrm{c}}, R=S=Q$, 由 $(15 \mathrm{a}) \sim(15 \mathrm{c}),(16)$ 及 $(17 \mathrm{a}) \sim$ $(17 \mathrm{c})$ 式可得到横观各向同性条件下准则(13)的材料 系数计算公式:

$$
\begin{gathered}
a_{0}=3\left(\frac{1}{2}\right)^{2 / 3}\left[\left(\frac{Y}{\sigma_{3}^{\mathrm{t}}}\right)^{3}+\left(\frac{Y}{\sigma_{3}^{\mathrm{c}}}\right)^{3}\right]^{2 / 3}, \\
a_{0}+a_{3}=6\left(\frac{1}{2}\right)^{2 / 3}\left[\left(\frac{Y}{\sigma_{0}^{\mathrm{t}}}\right)^{3}+\left(\frac{Y}{\sigma_{0}^{\mathrm{c}}}\right)^{3}\right]^{2 / 3}, \\
a_{4}=a_{5}=\frac{Y^{2}}{Q^{2}}, \quad a_{6}=\frac{Y^{2}}{T^{2}}, \\
17 b_{0}+b_{3}=-\frac{9}{2}\left[\left(\frac{Y}{\sigma_{0}^{\mathrm{t}}}\right)^{3}-\left(\frac{Y}{\sigma_{0}^{\mathrm{c}}}\right)^{3}\right], \\
2 b_{0}+16 b_{3}=-\frac{9}{2}\left[\left(\frac{Y}{\sigma_{3}^{\mathrm{t}}}\right)^{3}-\left(\frac{Y}{\sigma_{3}^{\mathrm{c}}}\right)^{3}\right] .
\end{gathered}
$$

对于平面应力状态 $\left(\sigma_{x x}, \sigma_{y y}, \sigma_{x y}\right)$, 准则(13)可以 简化为

$$
\begin{aligned}
\Phi= & {\left[\frac{1}{6}\left(a_{2}+a_{3}\right) \sigma_{x x}^{2}-\frac{a_{3}}{3} \sigma_{x x} \sigma_{y y}+\frac{1}{6}\left(a_{1}+a_{3}\right) \sigma_{y y}^{2}+a_{6} \sigma_{x y}\right]^{3 / 2} } \\
& -\left\{5\left(b_{1} S_{x}^{3}+b_{2} S_{y}^{3}+b_{3} S_{z}^{3}\right)+4\left(b_{1}+b_{2}+b_{3}\right) S_{x} S_{y} S_{z}\right\} \\
= & Y^{3} .
\end{aligned}
$$

特别地, 当 $\sigma_{x y}=0$ 时

$$
\begin{aligned}
\Phi= & {\left[\frac{1}{6}\left(a_{2}+a_{3}\right) \sigma_{x x}^{2}-\frac{a_{3}}{3} \sigma_{x x} \sigma_{y y}+\frac{1}{6}\left(a_{1}+a_{3}\right) \sigma_{y y}^{2}\right]^{3 / 2} } \\
& -\left\{5\left(b_{1} S_{x}^{3}+b_{2} S_{y}^{3}+b_{3} S_{z}^{3}\right)+4\left(b_{1}+b_{2}+b_{3}\right) S_{x} S_{y} S_{z}\right\} \\
= & Y^{3},
\end{aligned}
$$

其中, $S_{x}=\left(2 \sigma_{x x}-\sigma_{y y}\right) / 3, S_{y}=\left(2 \sigma_{y y}-\sigma_{x x}\right) / 3, S_{z}=-\left(\sigma_{x x}+\sigma_{y y}\right) / 3$.

材料常数 $a_{k}(k=1, \cdots, 3), b_{k}(k=1, \cdots, 3)$ 由(15a) (15c)和(17a) (17c)式确定. 对于板料, 厚度方向的力 学性能 $\left(\sigma_{3}^{\mathrm{t}}, \sigma_{3}^{\mathrm{c}}\right)$ 通常不易获得, 可用 $x$ - $y$ 平面内的双
向等拉及双向等压试验代替. 将 $\sigma_{x x}=\sigma_{y y}=\sigma_{\mathrm{bi}}^{\mathrm{t}}$ 及 $\sigma_{x x}=\sigma_{y y}=-\sigma_{\mathrm{bi}}^{\mathrm{c}}$ 带入准则(13)可得

$$
\begin{gathered}
a_{1}+a_{2}=6\left(\frac{1}{2}\right)^{2 / 3}\left[\left(\frac{Y}{\sigma_{\mathrm{bi}}^{\mathrm{t}}}\right)^{3}+\left(\frac{Y}{\sigma_{\mathrm{bi}}^{\mathrm{c}}}\right)^{3}\right]^{2 / 3}, \\
b_{1}+b_{2}+16 b_{3}=\frac{9}{2}\left[\left(\frac{Y}{\sigma_{\mathrm{bi}}^{\mathrm{t}}}\right)^{3}-\left(\frac{Y}{\sigma_{\mathrm{bi}}^{\mathrm{c}}}\right)^{3}\right] .
\end{gathered}
$$

\section{2 模型的评估}

本节将详细讨论屈服准则(13)用于不同材料时 屈服面的形状. 为方便起见, 将讨论限定在横观各向 同性材料上 $(x-y$ 平面内为各向同性). 考虑材料各向 异性系数及拉-压异性系数的不同，一共有 9 种不同 类型的材料, 分为 3 类.

1) 各向同性材料: 拉-压对称 $\left(k=\sigma_{\mathrm{t}} / \sigma_{\mathrm{c}}=1\right)$ 或拉-压 非对称 $(k \neq 1)$, 定义为材料 A.

2) 横观各向同性材料: 面内强度低于厚度方向 强度, 拉-压对称 $(k=1)$ 或拉-压非对称 $(k \neq 1)$, 定义为 材料 B.

3) 横观各向同性材料: 面内强度高于厚度方向 强度, 拉-压对称 $(k=1)$ 或拉-压非对称 $(k \neq 1)$, 定义为 材料 C.

其中各向同性材料 $\mathrm{A}$ 的选取是出于对照的考虑. 回顾前面关于横观各向同性材料的讨论，设 $\sigma_{3}^{\mathrm{t}} / \sigma_{0}^{\mathrm{t}}=\alpha, \sigma_{0}^{\mathrm{t}} / \sigma_{0}^{\mathrm{c}}=\sigma_{3}^{\mathrm{t}} / \sigma_{3}^{\mathrm{c}}=k$, 则不包含剪应力 时, 准则(13)的系数可由(18a)，(18b)和(20a), (20b)式 计算得到

$$
\begin{gathered}
a_{0}=6\left(\frac{1}{2}\right)^{2 / 3}\left[\left(\frac{1}{\alpha}\right)^{3}+\left(\frac{k}{\alpha}\right)^{3}\right]^{2 / 3}, \\
a_{0}+a_{3}=6\left(\frac{1}{2}\right)^{2 / 3}\left(1+k^{3}\right)^{2 / 3}, \\
17 b_{0}+b_{3}=-\frac{9}{2}\left(1-k^{3}\right), \\
2 b_{0}+16 b_{3}=-\frac{9}{2}\left[\left(\frac{1}{\alpha}\right)^{3}-\left(\frac{k}{\alpha}\right)^{3}\right] .
\end{gathered}
$$

表 1 所示为计算得到的 9 种理论材料的屈服准则 系数. 
表 1 三种理论材料 A, B, C 的屈服准则材料系数

\begin{tabular}{|c|c|c|c|c|c|c|c|c|c|}
\hline \multirow{2}{*}{ Parameter } & \multicolumn{3}{|c|}{ Material A } & \multicolumn{3}{|c|}{ Material B } & \multicolumn{3}{|c|}{ Material C } \\
\hline & $k=1$ & $k=5 / 4$ & $k=3 / 4$ & $k=1$ & $k=5 / 4$ & $k=3 / 4$ & $k=1$ & $k=5 / 4$ & $k=3 / 4$ \\
\hline$a_{0}$ & 3 & 3.89 & 2.39 & 1.92 & 2.49 & 1.53 & 5.33 & 6.92 & 4.25 \\
\hline$a_{3}$ & 3 & 3.89 & 2.39 & 4.08 & 5.29 & 3.25 & 0.67 & 0.86 & 0.53 \\
\hline$b_{0}$ & 0 & 0.24 & -0.15 & 0 & 0.25 & -0.15 & 0 & 0.22 & -0.13 \\
\hline$b_{3}$ & 0 & 0.24 & -0.15 & 0 & 0.11 & -0.07 & 0 & 0.61 & -0.37 \\
\hline
\end{tabular}

图 4(a) (c)分别为拉压比 $k=1,5 / 4,3 / 4$ 时, A, B, C 三种材料的屈服面在 $x-z$ 平面上的投影.

图 5(a) (c)分别为 A, B, C 三种材料不同拉压比 的屈服面在 $\pi$ 平面上的投影. 可以发现, 本文提出的 屈服准则是通过屈服面在某一方向的“拉伸”和相反 方向的“收缩”, 而不是通过 $\pi$ 平面上屈服轨迹的平移 (对比图 1)来包含 SD 效应的. 因此, 准则(13)克服了 Hosford 屈服准则 ${ }^{[7]}$ 的缺陷, 理论上更完备.

\section{3 应用举例}

前面详细讨论了本文提出的屈服准则((13)式)应 用到不同类型材料时屈服面的形状. 然而, 理论是否 正确, 还需要真实材料的检验. 本节将建立的准则应 用到两种拉-压非对称材料, 以评估模型的预测能力.

\subsection{Cu-Al-Be 记忆合金}

Bouvet 等人 ${ }^{[32]}$ 采用薄壁圆管试件内压和轴向拉 伸( 压缩) 以及立方块试件双向压缩的试验方法, 获 得了 $\mathrm{Cu}-\mathrm{Al}-\mathrm{Be}$ 记忆合金在平面应力状态下的初始屈 服轨迹. 具体试验数据见参考文献[26]. 图 6 所示为 $\mathrm{Cu}-\mathrm{Al}-\mathrm{Be}$ 记忆合金与 $0.2 \%$ 塑性应变对应的初始屈服 轨迹(试验数据由圆点表示). $\mathrm{Cu}-\mathrm{Al}-\mathrm{Be}$ 记忆合金的塑 性各向异性较弱, 但具有很强的拉-压非对称性, 其 单向压缩屈服强度比拉伸屈服强度高 20\%左右. 图 6 同时绘出了屈服准则((22)式)的理论屈服轨迹. 准则 的材料系数由试验数据根据 (15a), (15b), (17a), (17b) 和(23a), (23b) 式确定. 计算得到的准则系数见表 2 . 可以发现, 除了个别试验点外, 理论屈服轨迹与试验 数据吻合得很好. 可见, 本文提出的准则能很好地描 述 $\mathrm{Cu}-\mathrm{Al}-\mathrm{Be}$ 记忆合金屈服行为的拉-压非对称性.

\section{$3.2 \mathrm{Ni}_{3} \mathrm{Al}$ 基金属间化合物 IC10 合金}

IC10 合金是我国最新研制的 $\mathrm{Ni}_{3} \mathrm{Al}$ 基高温合金,
可用于制造航空发动机浴轮导向叶 ${ }^{[33]}$. 试验表明, IC10 合金在高温下具有强度高、延展性好等一系列 优良性能 ${ }^{[34]}$. IC10 合金采用定向凝固方式制取，是一 种柱晶组织 (有明显的 $<001>$ 生长方向), 其力学行为 呈明显的各向异性. 另外, $\mathrm{Li}_{2}$ 型长程有序金属间化合 物 $\mathrm{Ni}_{3} \mathrm{Al}$ 由于其不满足 $\mathrm{Schmid}$ 定律的晶体滑移而具 有显著的拉-压非对称性 ${ }^{[14,15]}$. 因此, IC10 合金的屈 服行为同时具有塑性各向异性及拉-压非对称性. 为 了研究 IC10 合金复杂的屈服行为, 作者曾对其进行 了双向拉伸 ${ }^{[35]}$ 及双向压缩试验 ${ }^{[36]}$, 获得了与 $0.2 \%$ 塑 性应变对应的初始屈服轨迹(见图 7, 试验数据由圆 点表示, 其中 $x$ 方向为定向凝固方向, $y$ 方向为横向). 试验结果表明, IC 10 合金的屈服轨迹关于双向等拉线 呈明显的非对称性，这与其塑性各向异性对应; 另外, IC10 合金的屈服轨迹还表现出拉-压非对称性, 其定 向凝固方向拉伸屈服强度大于压缩屈服强度, 横向 则呈相反的规律. 图 7 同时绘出了本文提出的屈服准 则(22)的预测结果. 准则的材料系数由试验数据根据 (15a)，(15b)，(17a)，(17b)和(23a)，(23b) 式计算得到 (结果见表 2). 可以发现, 理论预测与试验数据吻合 得很好, 本文提出的屈服准则能很好地描述 IC10 合 金的塑性各向异性及拉-压非对称性.

\section{4 总结}

巧妙地构造了一个与静水压力无关、能描述材料 拉-压非对称性的宏观各向同性屈服准则. 该准则只 包含一个能用材料拉伸和压缩屈服强度确定的材料 常数 $c$. 当材料拉-压对称时 $(c=0)$, 该准则退化为 von Mises 屈服准则. 其次, 利用 Cazacu 的广义应力偏量 不变量 $J_{2}^{0}$, 将其推广到正交各向异性条件, 建立了 一个能同时描述材料塑性各向异性及拉-压非对称性 的宏观屈服准则. 该准则能用于 3-D 应力状态, 只包 含 6 个描述材料塑性各向异性及 3 个拉-压非对称性 

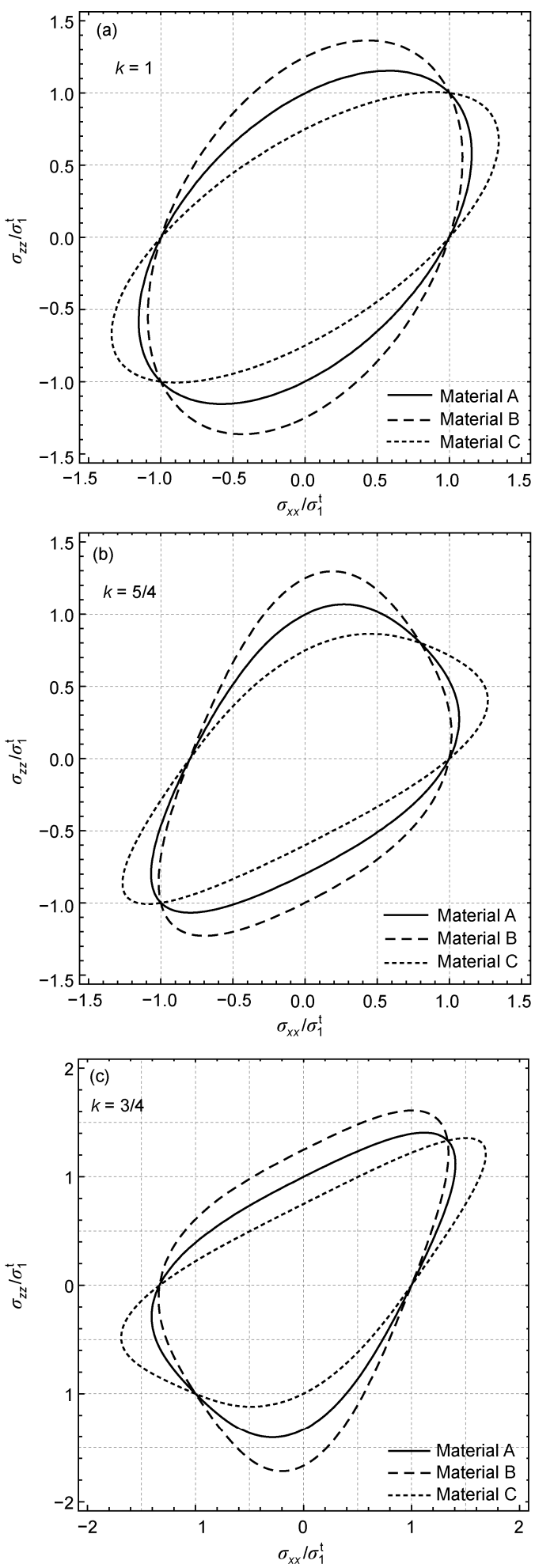

图 $4 \mathrm{~A}$ (各向同性), B, C (横观各向同性) 3 种理论材料在平 面应力状态下的屈服轨迹. $x$ 为面内方向, $z$ 为厚度方向 (a) 拉-压同性 $(k=1)$; (b) 拉伸屈服强度大于压缩屈服强度 $(k=5 / 4)$; (c) 拉伸屈服强度小于压缩屈服强度 $(k=3 / 4)$
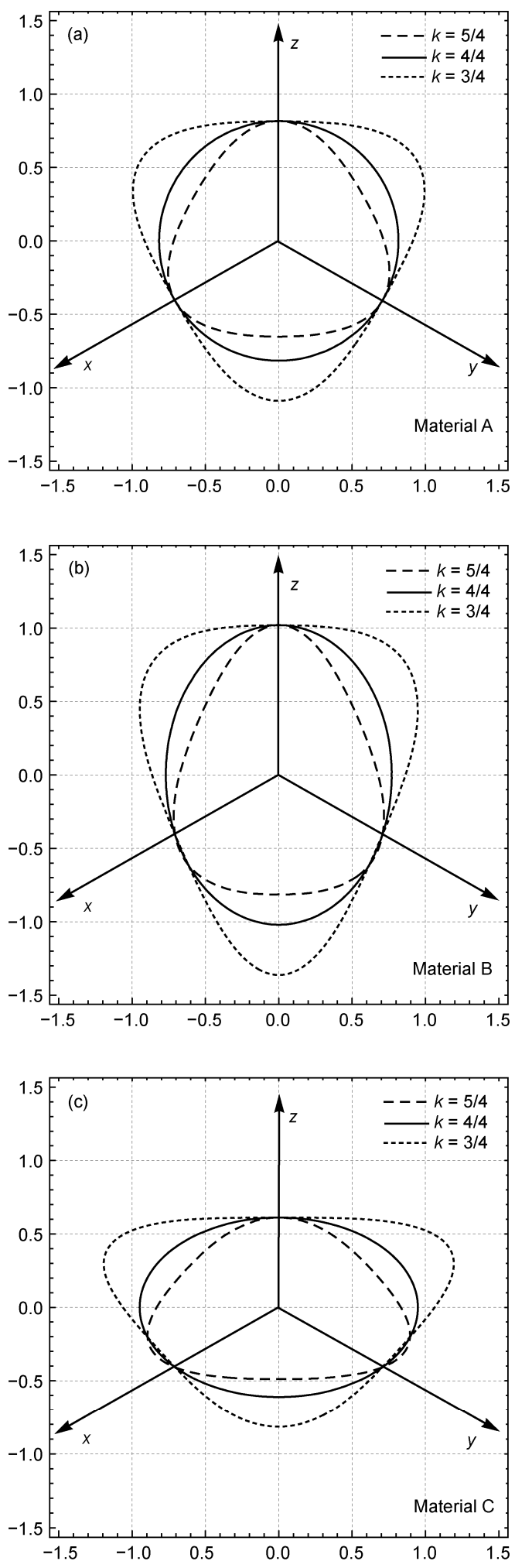

图 $5 \mathrm{~A}$ (各向同性), B, C (横观各向同性) 3 种材料的屈服面 在 $\boldsymbol{\pi}$ 平面上的投影. $\boldsymbol{x}, \boldsymbol{y}$ 为面内方向; $z$ 为厚度方向 (a) 材料 $\mathrm{A}$; (b) 材料 B; (c) 材料 C 


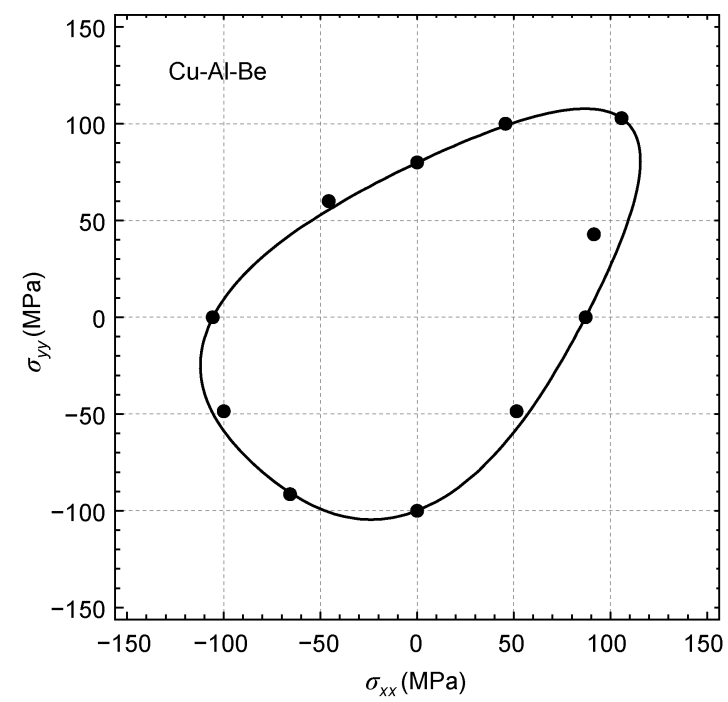

图 $6 \mathrm{Cu}-\mathrm{Al}-\mathrm{Be}$ 记忆合金试验屈服点与理论屈服轨迹的比 较(试验数据来自 Laydi 和 Lexcellent ${ }^{[26]}$ )

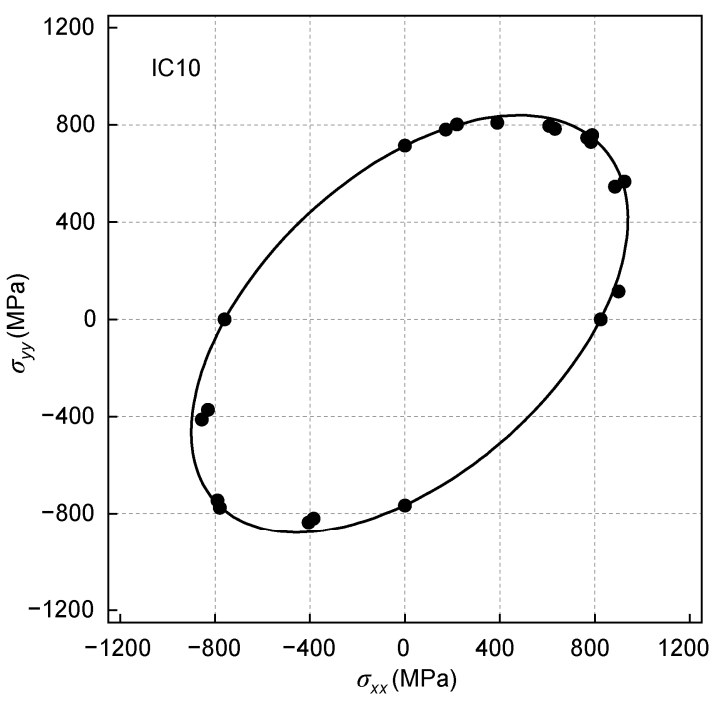

图 $7 \mathrm{Ni}_{3} \mathrm{AI}$ 基金属间化合物 IC10 合金试验屈服点与理论屈 服轨迹的比较

表 $2 \mathrm{Cu}-\mathrm{Al}-\mathrm{Be}$ 记忆合金和 IC10 合金的屈服准则材料系数

\begin{tabular}{lcccccrr}
\hline & $a_{1}$ & $a_{2}$ & $a_{3}$ & $b_{1}$ & \multicolumn{1}{c}{$b_{2}$} & $b_{3}$ & $Y(\mathrm{MPa})$ \\
\hline $\mathrm{Cu}-\mathrm{Al}-\mathrm{Be}$ & 3.291 & 2.468 & 2.617 & -0.102 & -0.160 & -0.182 & 87.14 \\
$\mathrm{IC} 10$ & 3.913 & 2.976 & 3.570 & 0.083 & -0.087 & 0.014 & 825.68 \\
\hline
\end{tabular}

的材料常数. 给出了准则材料常数的计算公式, 所有 常数都能通过简单力学试验确定. 当材料拉-压对称 时, 该准则退化为 Hill (1948) 屈服准则. 因此, 该准 则可看作 Hill (1948)准则向拉-压非对称材料的推广. 对 $\mathrm{Cu}-\mathrm{Al}-\mathrm{Be}$ 记忆合金及 $\mathrm{Ni}_{3} \mathrm{Al}$ 基金属间化合物 IC10 合金的应用表明, 本文提出的屈服准则能很好地描 述这两种合金的塑性各向异性及拉-压非对称性. 相 比其他学者提出的能描述材料 SD 效应的屈服准则, 本文提出的准则形式简单，更便于实际应用.

\section{附录 A Cazacu (2004) 屈服准则}

3-D 应力状态下, Cazacu (2004)屈服准则可以表 示为

$$
\left(J_{2}^{0}\right)^{3 / 2}-c J_{3}^{0}=\tau_{\mathrm{Y}}^{3},
$$

其中 $\tau_{\mathrm{Y}}$ 为剪切屈服强度, $c$ 为材料常数; $J_{2}^{0}, J_{3}^{0}$ 分别 为应力偏量 $S$ 第二、第三不变量 $J_{2}, J_{3}$ 在正交各向异 性条件下的推广, 具体表达式为

$$
\begin{aligned}
J_{2}^{0}= & \frac{a_{1}}{6}\left(\sigma_{y y}-\sigma_{z z}\right)^{2}+\frac{a_{2}}{6}\left(\sigma_{z z}-\sigma_{x x}\right)^{2} \\
& +\frac{a_{3}}{6}\left(\sigma_{x x}-\sigma_{y y}\right)^{2}+a_{4} \sigma_{y z}^{2}+a_{5} \sigma_{z x}^{2} \\
& +a_{6} \sigma_{x y}^{2}, \\
J_{3}^{0}= & \frac{1}{27}\left(b_{1}+b_{2}\right) \sigma_{x x}^{3}+\frac{1}{27}\left(b_{3}+b_{4}\right) \sigma_{y y}^{3} \\
+ & \frac{1}{27}\left[2\left(b_{1}+b_{4}\right)-b_{2}-b_{3}\right] \sigma_{z z}^{3} \\
- & \frac{1}{9}\left(b_{1} \sigma_{y y}+b_{2} \sigma_{z z}\right) \sigma_{x x}^{2}-\frac{1}{9}\left(b_{3} \sigma_{z z}+b_{4} \sigma_{x x}\right) \sigma_{y y}^{2} \\
- & \frac{1}{9}\left[\left(b_{1}-b_{2}+b_{4}\right) \sigma_{x x}+\left(b_{1}-b_{3}+b_{4}\right) \sigma_{y y}\right] \sigma_{z z}^{2} \\
+ & \frac{2}{9}\left(b_{1}+b_{4}\right) \sigma_{x x} \sigma_{y y} \sigma_{z z} \\
- & \frac{\sigma_{y z}^{2}}{3}\left[\left(b_{6}+b_{7}\right) \sigma_{x x}-b_{6} \sigma_{y y}-b_{7} \sigma_{z z}\right] \\
- & \frac{\sigma_{z x}^{2}}{3}\left[2 b_{9} \sigma_{y y}-b_{8} \sigma_{z z}-\left(2 b_{9}-b_{8}\right) \sigma_{x x}\right]
\end{aligned}
$$




$$
\begin{aligned}
& -\frac{\sigma_{x y}^{2}}{3}\left[2 b_{10} \sigma_{z z}-b_{5} \sigma_{y y}-\left(2 b_{10}-b_{5}\right) \sigma_{x x}\right] \\
& +2 b_{11} \sigma_{y z} \sigma_{z x} \sigma_{x y},
\end{aligned}
$$

式中, $x, y, z$ 分别与材料 3 个各向异性主轴重合. 各向 同性条件下, 系数 $a_{k}(k=1, \cdots, 6), b_{k}(k=1, \cdots, 11)$ 都 等于 $1, J_{2}^{0}, J_{3}^{0}$ 分别退化为 $J_{2}, J_{3}$.

\section{参考文献}

1 Banabic D, Cazacu O, Barlat F, et al. Advances in anisotropy and formability. Int J Mater Form, 2010, 3: 165-189

2 Yang H, Fan X G, Sun Z C, et al. Recent developments in plastic forming technology of titanium alloys. Sci China Tech Sci, 2011, 54: 490-501

3 俞茂宏. 强度理论百年总结. 力学进展, 2004, 34: 529-560

4 Hill R. A theory of the yielding and plastic flow of anisotropic metals. Proc Soc London A, 1948, 193: 281-297

5 Hill R. Theoretical plasticity of textured aggregates. Math Proc Camb Phil Soc, 1979, 85: 179-191

6 Hill R. A user-friendly theory of orthotropic plasticity in sheet metals. Int J Mech Sci 1993, 35: 19-25

7 Hosford W F. Texture strengthening. Metals Eng Quart, 1966, 6: 13-19

8 Hosford W F. A generalized isotropic yield criterion. J Appl Mech, 1972, 39: 607-609

9 Barlat F, Lian J. Plastic behavior and stretchability of sheet Metals. Part I: Yield function for orthotropic sheets under plane stress conditions. Int J Plasticity, 1989, 5: 51-66

10 Barlat F, Lege D J, Brem J C. A six-component yield function for anisotropic materials. Int J Plasticity, 1991, 7: 693-712

11 Barlat F, Becker R C. Yielding description of solution strengthened aluminum alloys. Int J Plasticity, 1997, 13: 385-401

12 Hosford W F, Allen T J. Twining and directional slip as a cause for strength differential effect. Met Trans, 1973, 4: 1424-1425

13 Hosford W F. The Mechanics of Crystals and Textured Polycrystals. New York: Oxford University Press, 1993

14 Ezz S S, Pope D P, Paidar V. The tension/compression flow stress asymmetry in $\mathrm{Ni}_{3}$ (A1, Nb) single crystals. Acta Metall, 1982, 30 : $921-926$

15 Umakoshi Y, Pope D P, Vitek V. The asymmetry of the flow stress in $\mathrm{Ni}_{3}$ (Al Ta) single crystals. Acta Metall, 1984, 32: 449-456

16 Cazacu O, Barlat F. A criterion for description of anisotropy and yield differential effects in pressure-insensitive metals. Int $\mathrm{J}$ Plasticity, 2004, 20: 2027-2045

17 Cazacu O, Barlat F. Generalization of Drucker's yield criterion to orthotropy. Math Mech Solids, 2001, 6: 613-630

18 Cazacu O, Barlat F. Application of representation theory to describe yielding of anisotropic aluminum alloys. Int J Eng Sci, 2003, 41: $1367-1385$

19 Verma R K, Kuwabara T, Chung K, et al. Experimental evaluation and constitutive modeling of non-proportional deformation for asymmetric steels. Int J Plasticity, 2011, 27: 82-101

20 Steglich D, Brocks W, Bohlen J, et al. Modeling direction-dependent hardening in magnesium sheet forming simulations. Int J Mater Form, 2011, 4: 243-253

21 Barlat F, Gracio J J, Lee M G, et al. An alternative to kinematic hardening in classical plasticity. Int J Plasticity, 2011, 27: 1309-1327

22 Mekonen M N, Steglich D, Bohlen J, et al. Mechanical characterization and constitutive modeling of Mg alloy sheets. Mater Sci Eng A, 2012, 540: $174-186$

23 Andar M O, Kuwabara T, Steglich D. Material modeling of AZ31 Mg sheet considering variation of $r$-values and asymmetry of the yield locus. Mater Sci Eng A, 2012, 549: 82-92

24 Cazacu O, Plunkett B, Barlat F. Orthotropic yield criterion for hexagonal close packed metals. Int J Plasticity, 2006, 22: 1171-1194

25 Plunkett B, Cazacu O, Barlat F. Orthotropic yield criteria for description of the anisotropy in tension and compression of sheet metals. Int $\mathbf{J}$ Plasticity, 2008, 24: 847-866

26 Laydi M R, Lexcellent C. Yield criteria for shape memory materials: Convexity conditions and surface transport. Math Mech Solids, 2010, 15: 165-208

27 Hershey A V. The plasticity of an isotropic aggregate of anisotropic face centered cubic crystals. J Appl Mech Trans ASME, 1954, 21: 241-249

28 俞茂宏, 何丽南, 宋凌宇. 双剪应力强度理论及其推广. 中国科学 A 辑: 数学 物理学 天文学, 1961, (12): 1113-1120

29 俞茂宏, 何丽南, 刘春阳. 广义双剪应力屈服准则及其推广. 科学通报, 1992, (2): 182-185

30 姚仰平, 路德春, 周安楠, 等. 广义非线性强度理论及其变换应力空间. 中国科学 $\mathrm{E}$ 辑: 工程科学 材料科学, 2004, 34: 1283-1299

31 Drucker D C. Relation of experiments to mathematical theories of plasticity. J Appl Mech, 1949, 16: 349-357

32 Bouvet $\mathrm{C}$, Calloch S, Lexcellent C. Mechanical behavior of a Cu-Al-Be shape memory alloy under multiaxial proportional and nonproportional loadings. ASME J Eng Mater Technol, 2002, 124: 112-124

33 赵希宏, 黄朝晖, 谭永宁, 等. 新型 $\mathrm{Ni}_{3} \mathrm{Al}$ 基定向高温合金 IC10. 航空材料学报, 2006, 26: 20-24

34 张宏建, 温卫东, 崔海涛, 等. 不同温度下 IC10 合金的本构关系. 航空学报, 2008, 29: 499-504

35 陈雷, 温卫东, 崔海涛. 双向应力状态下 IC10 高温合金的屈服行为研究. 航空学报, 2012, 33: 77-84

36 Chen L, Wen W D, Cui H T. Yielding description for a Ni $\mathrm{N}_{3} \mathrm{Al}$ based intermetallic alloy. Mater Des, 2012, 41: 192-197 